Relations industrielles

Industrial Relations

\title{
La grève, phénomène de civilisation par Robert Gubbels. Etudes d'économie sociale. Université libre de Bruxelles. Institut de sociologie Solvay. Bruxelles, 1962. 334 pages.
}

\section{Gérard Dion}

Volume 18, numéro 3, juillet 1963

URI : https://id.erudit.org/iderudit/1021411ar

DOI : https://doi.org/10.7202/1021411ar

Aller au sommaire du numéro

\section{Éditeur(s)}

Département des relations industrielles de l’Université Laval

\section{ISSN}

0034-379X (imprimé)

1703-8138 (numérique)

Découvrir la revue

Citer ce compte rendu

Dion, G. (1963). Compte rendu de [La grève, phénomène de civilisation par Robert Gubbels. Etudes d'économie sociale. Université libre de Bruxelles. Institut de sociologie Solvay. Bruxelles, 1962. 334 pages.] Relations industrielles / Industrial Relations, 18(3), 429-430. https://doi.org/10.7202/1021411ar

Tous droits réservés @ Département des relations industrielles de l’Université Laval, 1963
Ce document est protégé par la loi sur le droit d'auteur. L’utilisation des services d'Érudit (y compris la reproduction) est assujettie à sa politique d'utilisation que vous pouvez consulter en ligne.

https://apropos.erudit.org/fr/usagers/politique-dutilisation/ 
de souligner la qualité particulière d'un article de $M$. Edward $G$. Bennion sur le budget de copital et la théorie des jeux. L'introduction des outeurs, Bock et Holstein, à cette partie qui analyse la planification des immobilisations, remplit bien, croyonsnous, I'objet d'un tel texte.

Nous reconnaissons le grand avantage de trouver sous une même couverture vingt excellents orticles parus assez récemment sur l'administration de la production.

\section{Bertrand BELZILE}

La grève, phénomène de civilisation par Robert Gubbels. Etudes d'économie sociale. Université libre de Bruxelles. Institut de sociologie Solvay. Bruxelles, 1962. 334 pages.

Cette étude est le fruit d'une recherche et d'une analyse de ce phénomène économico-social difficile à comprendre et difficile à admettre pour les profanes. Ils ne sont pas rares ceux qui voudraient voir l'Etat prohiber la grève mais qui ne savent pas trop comment remplacer ce moyen violent de régler les conflits. C'est ce qui faisait dire naguère à Roger Roch: « Si nous ne craignions de paraître paradoxal, nous dirions volontiers: ou point de vue social, la contestation du droit de grève est plus dangereuse que l'exercice de ce droit.»

L'outeur o divisé son ouvrage en quatre parties qu'il fait suivre de conclusions générales: les données de fait; les données économiques et statistiques; les données juridiques; les données sociologiques.

Dons les données de fait, il présente assez sommairement des études de cas qui couvient différentes grèves dans des secteurs particuliers soit deux grèves d'entreprises pour l'exercice du droit syndical, deux grèves de métallurgistes, une grève dans un service public, une grève générale sur le plan du secteur, une autre sur le plon régional et enfin deux grèves généralisées sur la plan national. Les types qu'il a choisis donnent une assez bonne idée des différences qui peuvent se rencontrer et des solutions qui peuvent être apportées dons le règlement.

L'onalyse statistique porte sur les grèves qui ont eu lieu en Belgique depuis le ler janvier 1954 au 31 décembre 1960. Nous ne savons pos si cette période est bien représentotive. Nous avons été particulièrement intéressé oux discussions préliminaires sur lo validité des statistiques de grèves et sur les avantages et les inconvénients d'utiliser soit le nombre de conflits ou le nombre de journées de travail perdues dans les grèves. II est évident que dans les pays où la grève générole n'existe à peu près pos et où l'on n'o que des grèves d'étoblissement, comme c'est le cas aux Etats-Unis et au Canada, on ne soit pas porté à utiliser les mêmes façons de calculer. On peut le regretter, car il est difficile par la suite de faire des comparaisons sur le plan international.

Dans la partie consacrée aux données juridiques, l'auteur étudie comment en Belgique sont organisées la prévention et la solution des confiits du travail, il expose la législation et la réglementation d'intérêt public et enfin le régime juridique de la grève. II ne le fait pas tant avec une préoccupotion de juriste qu'avec celle d'un sociologue. Cette partie nous apparait comme un excellent résumé des aspects juridiques de la grève en Belgique.

Ce qui nous a intéressé davantage, c'est lo partie de l'ouvroge consacrée aux données sociologiques. Elle est la plus longue et comprend trois sections, chacune divisée en plusieurs chapitres: typologie de la grève, le contexte d'une grève, les acteurs d'une grève. C'est ici que le lecteur non initié peut commencer à saisir comment la grève est un phénomène de civilisation. Mais il le verra bien dans les conclusions où l'auteur essaie de répondre à la question «pourquoi une sociologie de la grève? ». II commence par faire le bilan des notions de grève communément occeptées et des notions contestées. Il tire ensuite les notions qui résultent de so propre étude pour terminer en présentant un essai de définition et de classification des grèves.

On sera intéressé à connaitre sa longue définition de la grève: «la grève est un acte par lequel un groupe social manifeste à la fois sa solidarité interne et so désolidarisation par rapport au reste de la société; cette manifestation se traduit généralement, mais pas nécessairement, par un arrêt concerté du travail; le groupe social y recourt ofin d'exprimer une volonté, un mécontentement ou une opinion, dans le cas où il ne trouve plus d'autre moyen pour influencer les décisions à prendre en cette matière. 》D Définition longue, assez alambiquée. Pourquoi ovoir ajouté «dans les cas où il (le groupe social) ne trouve plus d'outre moyen... etc. \On élimine ainsi la grève systématique. Pour notre part, nous serions porté ò dire tout simplement: la grève est I'utilisation concertée de la prestation de travail comme moyen de pression pour ame- 
Relations IndUSTRIELLES, vol. 18 , No 3

ner les autres ò accepter des revendications ou ò régler un litige. L'essai de classification que fait l'auteur dénote un bel effort de synthèse, mais il pourrait être plus complet. On voit qu'il s'en tient surtout à l'expérience de son propre pays.

L'auteur, dans son ouvrage, foit montre d'une bonne érudition. II a pris connaissance de la plupart des travaux publiés sur le sujet et la bibliographie que contient un des annexes est assez vaste. Nous regrettons cependant que lui ait échappé une étude de Von Nell-Breuning intitulée $<\mathrm{Im}$ plications politiques de la grève » que publiait en 1956 la Revue de l'Action populaire. A notre sens, les considérations de l'éminent professeur allemand sont tout à fait fondamentales dans toute étude sur la grève.

La grève, phénomène de civilisation est un ouvrage sérieux qui enrichit la littérature sur un sujet oussi importont qui n'a pas fini d'attirer l'attention des sociologues, des économistes et des juristes.

Gérord DION

Stabilité du Personnel dans les Hôpitaux du Québec, Rapports no 1 et no 2, Conseil de Recherche de l'Ecole Supérieure d'Administration Hospitalière du Comité des Hôpitaux du Québec, miméographié 70 pages et 108 pages, 1963.

Le premier rapport analyse l'ancienneté du personnel, le second analyse les causes de départ des salariés à l'emploi des hôpitaux.

\section{Jacques ST-LAURENT}

The Anvil and the Plow, U.S. Department of Labor, Office of Information, Publications, and Reports, 1963, 302 pages.

L'histoire du Ministère du Travail américain rédigée à l'occosion de son cinquantième anniversaire. C'est un rappel des changements majeurs survenus dans le monde ouvrier américain depuis 1913 et de la façon dont le Ministère du Travail s'est odapté à une société en continuelle évolution.

\section{Jacques ST-LAURENT}

Labor in the United States, by Sanford Cohen. Charles E. Merrill Books, Inc., Columbus, Ohio. 1960. 675 pages.

Cet ouvrage veut être une introduction à l'étude des problèmes des relations du travail pour le bénéfice des étudiants et aussi des personnes engagées dans ce domaine. L'approche de l'auteur est interdisciplinaire. C'est pourquoi on $y$ trouvera de I'histoire, de la sociologie, du droit, de l'économique et des sciences politiques.

L'outeur, qui enseigne depuis longtemps cette matière, maîtrise bien son sujet et ne cherche pas à faire oeuvre scientifique. II a divisé son étude en sept parties: Lo main d'oeuvre oux Etats-Unis, le syndicalisme oux Etots-Unis, la négociation collective; I'analyse des solaires; la législation du travail; le problème de I'insécurité économique; conclusion. A la fin de chaque chapitre, on trouve une série de questions pour les étudionts et une très courte bibliographie. Comme on peut s'en rendre compte, tout est centré sur des données américaines. C'est un bon manuel pour étudiants américains bien qu'on puisse regretter que l'auteur n'ait pas songé à les aider à prendre conscience que les Etats-Unis ne sont pos à eux seuls l'univers. Comment, en effet, bien comprendre même les problèmes américains sans tenir compte de l'interdépendance des économies et de I'influence des idéologies étrangères?

Malgré cette restriction, nous considérons que l'auteur, dont les jugements sont pondérés et honnêtes, o rendu service aux étudiants et oux professeurs en mettant entre leurs mains un ouvrage acceptable.

Gérard DION

《Introduction to Social Welfare 》 (deuxième édition) par Walter A. Friedlander, Prentice Hall, Englewood Cliffs, N.J., 1961, 589 poges.

Cette révision complète et soignée tient compte des nombreux commentaires de centaines d'utilisateurs de la première édition parue en 1955. Ce volume explique comment les services et les concepts de bien-être social se sont développés à travers la tradition, l'expérience et le changement sociologique. II analyse dans une perspective historique et philosophique, le système complexe présent de bien-être social, ses principes, son cadre légal et ses effets sur les membres de notre société.

Selon l'auteur lui-même, ce livre s'adresse particulièrement à trois groupes de lecteurs: (1) ceux qui projettent de travailler, ou qui travaillent déjò, dans les domaines de l'assistance publique, de la récréation etc., (2) ceux intéressés d'une façon ou d'une autre aux études de service social, etc et 